\title{
Elastic Characteristics Calculation for Thin Coatings and Bodies with a Topocomposite Structure Considering Specific Deformation of Layered Bodies during Instrumented Indentation
}

\author{
Nikolay Voronin.* \\ Institute of Machine Science of RAS, Departure of Tribology, Mal. Kharitonevsky per. 4, Moscow 101990, Russian Federation \\ ${ }^{*}$ Corresponding author
}

\begin{abstract}
The present study addresses the elastic deformation of topocomposites with thin hard surface layers during instrumented indentation. We propose calculation techniques to establish the elastic characteristics of the topocomposite surface and the coating material, involving the results of instrumented indentation and a model based on the modified Hertz contact problem.
\end{abstract}

Keywords-instrumented indentation; thin coatings; topocomposites; elastic modulus

\section{INTRODUCTION}

Modern technologies for producing protective coatings have provided a range of small-thickness surface coatings to create efficient functional surfaces for a variety of purposes. One of the achieved goals is the ability to provide the required tribological characteristics. Predicting the working capacity and tribotechnical properties of friction surfaces with protective coatings is greatly facilitated by available data on the physical and mechanical characteristics of the coating material and of the surface layer system (topocomposite) as a whole. For the surfaces of tribotechnical design, hardness and elastic modulus are two key characteristics. The instrumental indentation method is widely used to evaluate the mechanical characteristics of the material employing the analysis and interpretation of the loading-indenter penetration diagram. Application of instrumental indentation for thin hardening coatings faces some considerable difficulties. This deficiency is gradually overcome by constant improvement of measurement techniques, the use of finite element modeling to describe the stress-strain state of the material during the indenter penetration, and the creation of new physical models for deforming surface layered systems and heterogeneous systems.

\section{OBJECTIVE}

The purpose of this study is to increase knowledge of the deformation mechanisms of the surface layered bodies (topocomposites) during instrumental indentation with pyramidal tips, and to create the methods for calculating the mechanical characteristics, including elastic moduli, of the surfaces hardened with thin protective hard coatings.

\section{SOlution Procedure}

The objective set in this work is achieved by applying the basic principles of the developed in [1-3] model of contact interaction of a spherical indenter with the elastic-plastic layered system surface simulating a solid surface hardened by a thin protective coating. The model is based on the modified Hertz problem, which takes into account the true diagram of the contact pressure in the sphere interaction with the hardened surface. The result of solving the mathematical model are the analytical expressions describing the changes in the deformation-force parameters of the contact and a number of effective characteristics of the layered system surface as a function of the indenter penetration depth, loading force, geometry of the contact, and the complex characterizing the relationship between the elastic and plastic properties of the layered system components. The obtained models for changing the loading parameters and effective characteristics are analyzed from the point of view of establishing the physical nature of the processes of deformation of surfaces with a topocomposite structure. The experimental load curve obtained by the instrumental indentation method, along with the composite hardness curve, is compared with the developed theoretical models, which makes it possible to calculate the required mechanical characteristics both of the surface as a whole and the components of the layered body separately.

\section{RESULTS AND DISCUSSION}

In accordance with our earlier analysis of the mechanics of the contact interaction of a hard spherical indenter with a planar topocomposite surface $[1,2]$, the relations connecting the depth of penetration $\delta_{c}$ of a spherical indenter into a layered space and the composite effective modulus $K_{c}$ of the topocomposite surface with the corresponding parameters characterizing a homogeneous material which is the substrate material of the topocomposite, are the following:

$$
\delta_{c}=\delta_{0} \times \Phi
$$




$$
K_{c}=K_{0} \times \Phi^{\frac{3}{2}} \text { or } E_{c}=E_{0} \times \Phi^{-\frac{3}{2}}
$$

where $\delta_{c}$ is the spherical indenter penetration into a perfectly elastic single-layer topocomposite; $\delta_{0}$ is the spherical indenter penetration into a homogeneous half-space having an elastic characteristic of the substrate material of the topocomposite; $\Phi$ is the elastic-geometric parameter: $\Phi=\sum_{i=0}^{m} A_{i} \cdot \bar{t}_{0}^{i} \cdot\left(\sum_{j=0}^{n} B_{j} \cdot \bar{t}_{0}^{j}\right)^{-1} ; \bar{t}_{0}=\frac{t_{0}}{a_{0}} ; A_{j}=f(K)$, $B_{j}=f(K) ; A_{1}, A_{2}, A_{3}, \ldots A_{i}, B_{1}, B_{2}, B_{3}, \ldots B_{j}$ are the coefficients of the two-point Padé approximants; ; $t_{0}$ - the thickness of the layer having properties of the coating material in a model twolayer half-space; $a_{0}$ - the radius of a contact indent calculated for a medium having elastic characteristics of the base material; $K=\frac{K_{1}}{K_{0}}$ is the effective contact modulus of a surface layered body; $K_{c}=\frac{1}{E_{c}}$ is the composite effective elastic modulus of a single-layer topocomposite, $K_{0}=\frac{1-\mu_{0}^{2}}{E_{0}}, K_{1}=\frac{1-\mu_{1}^{2}}{E_{1}}$ are the effective elastic constants of the single-layer topocomposite components: substrate and coating, respectively; $E_{1}$ is the Young's elastic modulus of the coating material, $\mu_{1}$ is the Poisson's ratio of the coating material; $E_{0}$ is the Young's elastic modulus of the substrate material, $\mu_{0}$ is the Poisson's ratio of the substrate material;

The study in [3] illustrates the possibility of transforming analytic expressions describing the mechanics of the contact interaction of a sphere with a two-layer solid body (a singlelayer topocomposite) into a dependence of the loading forces and hardness on the pyramidal indenter penetration, which is typical for the standard instrumented indentation procedure [4]. The analysis of the obtained theoretical dependences has shown that the hardness at indentation of the topocomposite surface over the entire depth of the surface layer with increasing penetration depth is characterized by three regions of existence of the parameters under study. Each region of the changes in hardness and loading force upon the penetration depth is described by analytic expressions with multiplier values defined for the given region:

- for hardness:

$$
H_{c}=H_{0} \cdot\left(\bar{\Phi}_{i}\right)^{\frac{1}{2}} \cdot(\Phi)^{-3 / 2}
$$

- for loading force (loading curve):

$$
P_{c}=H_{0} \cdot\left(\frac{s_{c}}{h}\right)^{2} \cdot h^{2} \cdot\left(\bar{\Phi}_{i}\right)^{\frac{3}{2}} \cdot(\Phi)^{-3 / 2}
$$

Where $\bar{\Phi}$ is the limiting elastic-geometric parameter with the following range of existence $1 \leq \bar{\Phi} \leq K^{2} Y^{2}$ for $0 \leq h \leq \infty ; \bar{\Phi}=M^{\mathrm{I}, \mathrm{II}, \mathrm{III}} \cdot(\Phi)^{3}$; at $M^{\mathrm{I}}=1$ :

$$
\begin{aligned}
& \frac{h}{a_{0}^{\kappa p}}=\frac{t_{0}}{a_{0}^{\kappa p}} \times(\Phi)^{3 / 2} ; \text { at } M^{\mathrm{II}}=\left(\frac{0.31}{T_{k}}\right)^{2}: \\
& \frac{h}{a_{0}^{k p}}=\frac{t_{0}}{a_{0}^{k p}} \times\left(\frac{0.31}{T_{k}}\right) \times(\Phi)^{3 / 2} ; \text { at } M^{\mathrm{III}}=Y^{2}: \\
& \frac{h}{a_{0}^{\kappa p}}=\frac{t_{0}}{a_{0}^{\kappa p}} \times \frac{(\Phi)^{3 / 2}}{K^{2 / 3}} ; \bar{t}_{0}^{\kappa p}=\frac{t_{0}}{a_{0}^{\kappa p}} ; \\
& T_{k}=\frac{1}{2} \cdot\left[\frac{3}{2} \cdot\left(1+\bar{t}_{0}^{2}\right)^{-1}-\left(1+\mu_{0}\right) \cdot\left(1-\bar{t}_{0} \cdot \operatorname{arctg}\left(\frac{1}{\bar{t}_{0}}\right)\right)\right] \\
& Y=\frac{H_{1}}{H_{0}}, H_{0}, H_{1} \text { is the hardness of the substrate material }
\end{aligned}
$$
and the coating, respectively; $s_{c}$ is the penetration depth of the pyramidal indenter corresponding to the load $P_{c}$ (more details on the derivation of the dependences and the notation of the parameters are provided in [3]).

Figure I plots the obtained above analytical dependences of the change in the surface hardness of a single-layer topocomposite and the penetration force on the pyramidal indenter penetration depth into the surface for one particular version of the topocomposite. The curves showing the loadpenetration depth dependence for homogeneous compact materials with characteristics corresponding to the substrate and coating materials are also given in the figure.

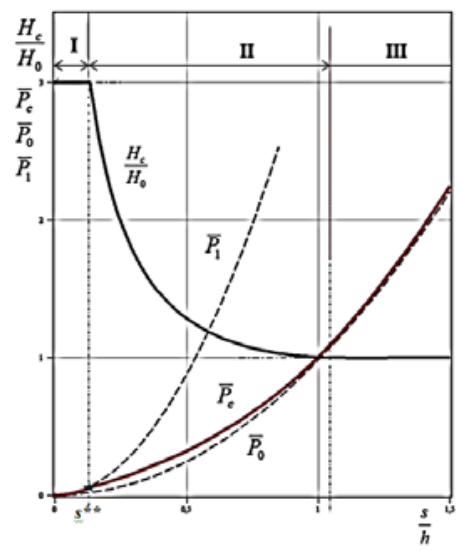

FIGURE I. THE DEPENDENCE OF THE PENETRATION FORCE AND COMPOSITE HARDNESS ON THE PENETRATION DEPTH OF THE VICKERS PYRAMID FOR THE TOPOCOMPOSITE WITH PARAMETERS $\mathrm{K}=0.5$ AND $\mathrm{Y}=3$ AND FOR THE HOMOGENEOUS COMPACT MATERIALS WITH CHARACTERISTICS CORRESPONDING TO THE SUBSTRATE AND COATING MATERIALS. 
For the topocomposite, (1) for the second region of the change in the surface hardness on the penetration depth will have the following form on rearrangement:

$$
P_{c}=H_{c} \cdot s_{c}^{2}=s_{c}^{2} \cdot H_{0} \cdot\left(\frac{0.31}{T_{k}}\right)
$$

The $T_{k}$ parameter depends only on the $\frac{h}{s_{c}}$ value and $K$. In the analysis of the hardness and loading curves during indentation in the second region, it is important that the composite hardness value in the second region of the coating thickness variation depends only on the substrate hardness and the elastic properties of the coating material. It means that it is not physically possible to predict the coating material hardness based on this curve. Therefore, we propose to use the loading dependence in the second region to determine the compositional and true values of the elastic modulus.

We consider two options of the calculation technique for the elastic modulus of the coating material according to the loading curve for a surface with a topocomposite structure.

The dependence of the load on the penetration depth during instrumental indentation in the substrate material without coating can be expressed through the hardness of the substrate:

$$
P_{0}=H_{0} \cdot s_{0}^{2}
$$

When load values are equal $P_{c}=P_{0}$, we obtain:

$$
\frac{s_{c}^{2}}{s_{0}^{2}}=\left(\frac{T_{k}}{0.31}\right)
$$

Where $T_{k}=f\left(K ; \frac{h}{s}\right)$ is the parameter depending on the effective contact modulus $K$ and the relative thickness of the coating. Taking into account the elastic properties of the indenter $K=\frac{K_{1}+K_{u}}{K_{0}+K_{u}}$, where $K_{u}=\frac{1-\mu_{u}^{2}}{E_{u}}$ is the effective elastic constant of the indenter material, $E_{u}, \mu_{u}$ are the Young's modulus and the Poisson's ratio of the indenter material, respectively.

The first calculating option for the coating elastic characteristics consists in comparing the array of values of the left-hand side of the equation (3) with the array of values of the right-hand side of the given equation. The values of the lefthand side of the equation are experimentally obtained from the penetration diagrams for a coated and uncoated surface, while the right-hand side array of values contains the data obtained theoretically from the penetration model for a number of effective contact module $\mathrm{K}$ discrete values for a layered body.
We can derive the Young's modulus of the coating material from the expression for the contact elastic modulus:

$$
E_{1}=\frac{1-\mu_{1}^{2}}{K_{0} \cdot K+K_{u}(K-1)}
$$

To implement the second calculating option for the coating elastic modulus, we rearrange the expression (2) to determine the penetration force through the theoretical hardness values for the topocomposite and the substrate:

$$
H_{c}=H_{0} \cdot\left(\frac{0.31}{T_{k}\{K\}}\right) ; H_{0}=\left[H_{0}\right] \cdot\left(\frac{0.31}{T_{k}\{K=1\}}\right)
$$

Where $\left[H_{0}\right]$ is the volume hardness of the substrate material As a result, we obtain

$$
\frac{P_{c}}{s_{c}^{2} \cdot\left[H_{0}\right]}=\left(\frac{0.31}{T_{k}\{K\}}\right) \cdot\left(\frac{0.31}{T_{k}\{K=1\}}\right)
$$

The left-hand side of expression (5) makes it possible to determine the array of values from the experimental penetration curves, while the right-hand side is calculated from the theoretical values calculated for a number of discrete values of the effective contact module $K$ of a layered body taking into account $K=1$. For the chosen value of $K$, the elastic modulus is determined by the formula (4). The proposed option makes considerably simplifies the procedure for calculating the effective elastic modulus and elastic modulus of the coating, since there is no need in the instrumental indentation of the substrate material, and the penetration curve for the substrate material does not require processing. In the calculations, the table elasticity modulus of the substrate material is used.

The results of calculating the elastic modulus of thin solid coatings obtained by vacuum ion-plasma methods using the techniques proposed in this study are in good agreement with the data available in the literature.

\section{ACKNOWLEDGMENT}

The work is done by financial support of Ministry of education and science RF Agreement №14.607.21.0166, 26.09.2017, Project RFMEFI60717X0166.

\section{REFERENCES}

[1] N.A. Voronin, "Modelling Kinetic Indentation in Topocomposite Surface of Elastic Rigid-plastic Solid Bodies". Advances in Computer Science Research. 2015, vol. 18, pp.993-997.

[2] N.A. Voronin, "The Influence of the Deformational Hardening of a Base Material on the Effective Characteristics of a Topocomposite". Journal of Machinery Manufacture and Reliability, 2014, vol. 43, No. 6, pp. 553-559.

[3] N. A. Voronin, "Composite and Real Hardnesses of Thin Coatings". Advanced Materials Research. 2012,vols. 560-561, pp. 803-808. 
[4] ISO 14577-4:2007. Metallic materials-Instrumented indentation test for hardness and materials parameters. Part 4: Test method for metallic and non-metallic coatings, 2007.-32p. 